\title{
CALOR ESPECÍFICO E DENSIDADE DA POLPA DE CAJÁ (Spondias lutea L.) COM DIFERENTES CONCENTRAÇÕES DE SÓLIDOS SOLÚVEIS SOB BAIXAS TEMPERATURAS
}

\author{
MARIO E. R. M. CAVALCANTI MATA ${ }^{1}$, MARIA E. M. DUARTE ${ }^{1}$, HELEN L. H. T. ZANINI ${ }^{2}$
}

RESUMO: A polpa de cajá tem sido exportada da região Nordeste para todo o Brasil, e uma das maneiras de conservá-la é processando-a e armazenado-a na forma congelada. Durante o processamento, existem dois fatores que favorecem a qualidade do produto a ser armazenado: a rapidez com que o produto é congelado e a adição de açúcar como forma de dificultar a ação microbiológica. $\mathrm{Na}$ linha de processo, algumas propriedades termofísicas, como o calor específico e a densidade, se alteram com as temperaturas negativas e com o teor de sólidos solúveis totais; dessa forma, para o dimensionamento adequado de máquinas e equipamentos, torna-se necessário conhecer essas propriedades, principalmente quando se operam equipamentos criogênicos, onde as temperaturas podem chegar a $-196{ }^{\circ} \mathrm{C}$. Neste trabalho, determinaram-se a densidade e o calor específico da polpa do cajá com quatro diferentes níveis de sólidos solúveis totais (natural da polpa; 20; 40 e 60\%) e temperaturas entre $-196{ }^{\circ} \mathrm{C} \mathrm{e}-18{ }^{\circ} \mathrm{C}$. A densidade foi obtida pela razão entre a massa da polpa e o seu volume. $\mathrm{O}$ volume foi determinado pelo método do deslocamento de massa (água) e o calor específico por meio de balanço de calor entre a massa da polpa, a massa do cilindro que contém a polpa e a massa do gelo. Conclui-se que a densidade da polpa de cajá aumenta em função da redução da temperatura e do aumento do teor de sólidos solúveis totais de 9 para $60^{\circ}$ Brix e que o calor específico da polpa diminui com a diminuição de temperatura e aumenta com a concentração de sólidos solúveis totais.

PALAVRAS-CHAVE: propriedades termofísicas, criogenia, congelamento.

\section{CAJÁ PULP (Spondias lutea L.) SPECIFIC HEAT AND DENSITY FOR DIFFERENT SOLUBLE SOLIDS CONCENTRATIONS AT LOW TEMPERATURES}

ABSTRACT: The cajá pulp has been exported from the Northeast area to all parts of Brazil, and one of the ways of conserving it is processing and storing it in the frozen form. During the processing, there are two factors that favor the quality of the product to be stored; the speed that the product is frozen and the addition of sugar as a form of hindering the microbiologic action. In the process line, some thermo-physics properties like the specific heat and the density change themselves according to the negative temperatures and the total soluble solids content. Then, it's necessary to know those properties mainly when cryogenic equipments are operated, where the temperatures can be $-196{ }^{\circ} \mathrm{C}$ to have the appropriate dimension of machines and equipment. Therefore, in this work, it was determined the cajá pulp density and the specific heat for 4 different levels of total soluble solids (natural of the pulp; 20; 40 and $60 \%$ ) and temperatures from -196 to $-18{ }^{\circ} \mathrm{C}$. The density was obtained by the rate between mass of the pulp and its volume. The volume was determined by the displacement of mass (water) and the specific heat was determined by the balance of heating among the mass of the pulp, the mass of the cylinder that contains the pulp and the mass of the ice. The results allow to conclude that: a) the cajá pulp density increases in function of the variation of the temperature from -18 to $-196{ }^{\circ} \mathrm{C}$, and also as a function of the increase of the total soluble solids content from 9 to $60^{\circ} \mathrm{Brix}$; b) the cajá pulp specific heat decreases according to the decrease of the temperature and it increases according to the concentration of total soluble solids.

KEYWORDS: thermo-physics properties, cryogenics, freezing.

\footnotetext{
${ }^{1}$ Prof.(a) Dr.(a), Depto. de Engenharia Agrícola, UFCG, Campina Grande - PB, mmata@ deag.ufcg.edu.br; elita@deag.ufcg.edu.br

${ }^{2}$ Doutoranda em Microbiologia, UNESP, Jaboticabal - SP, helenzanini@ yahoo.com.br

Recebido pelo Conselho Editorial em: 9-5-2003

Aprovado pelo Conselho Editorial em: 3-6-2005
} 


\section{INTRODUÇÃO}

O cajá é um fruto bastante apreciado em todo o Brasil, sendo mais consumido no Nordeste, na forma in natura e, nas outras regiões do País, na forma de polpa. Embora exista expectativa de desenvolvimento e expansão de seu cultivo, seus frutos são bastante perecíveis, havendo a necessidade de seu processamento para aumentar sua vida útil. Uma das maneiras mais utilizadas para armazenar a polpa do cajá, pela indústria alimentícia, é congelando-a imediatamente após a extração. A polpa também pode ser consumida pelas diferentes indústrias de transformação, como as de doces, sucos, refrescos e sorvetes.

O congelamento é um dos melhores métodos de armazenar um produto, com transformações mínimas, preservando seu valor nutritivo, sensorial, além de outros fatores responsáveis pela qualidade do produto. Nos alimentos congelados, a qualidade final está relacionada com as condições empregadas durante o processo de congelamento e com as condições de armazenamento (CIABOTTI, 2000).

AMER \& RUBIOLO (1998) relatam que o congelamento rápido de um alimento preserva sua qualidade, uma vez que seu uso leva à retenção de maior quantidade dos aromas voláteis que são perdidos durante o congelamento lento, além de ter menor fração de produto não-congelado.

Outra barreira importante para a conservação dos alimentos é a concentração de açúcares, principalmente pela sua capacidade de reduzir a atividade de água e, conseqüentemente, dificultar a ação microbiológica. Sua adição em sucos congelados também parece contribuir para a manutenção de algumas características sensoriais, tais como cor, aroma e sabor. Segundo GRUDA \& POSTOLSKI (1986), a adição de açúcar acentua o aroma e o sabor de muitas frutas, uma vez que evita a oxidação, durante o descongelamento. As proporções de açúcar utilizadas dependerão do destino final da polpa.

Assim, a velocidade do congelamento e a quantidade de açúcares são fatores importantes no planejamento e na construção de uma indústria de polpa de frutas, mas, para que se possa dimensionar adequadamente os equipamentos e otimizar os processos, é necessário conhecer as propriedades termofísicas do produto final, principalmente as mais básicas, como a massa específica e o calor específico sob temperaturas abaixo de $-18{ }^{\circ} \mathrm{C}$, pois, segundo KASAHARA et al. (1986) e SIEBEL (1982), essas propriedades estão sujeitas a mudanças durante o congelamento e se alteram em função da diminuição de temperatura e da quantidade de água presente no interior do produto. Esses parâmetros, por sua vez, influenciam na velocidade e no tempo de congelamento.

Como essas propriedades termofísicas, a temperaturas criogênicas (abaixo de $-70{ }^{\circ} \mathrm{C}$ ), não estão disponíveis na literatura, pois as técnicas de congelamento ultra-rápido são muito recentes, o presente trabalho teve como objetivo determinar a densidade e o calor específico da polpa de cajá com diferentes concentrações de sólidos solúveis totais (natural da polpa; 20; 40 e $60{ }^{\circ} \mathrm{Brix}$ ) a $-196{ }^{\circ} \mathrm{C}$; $180{ }^{\circ} \mathrm{C} ;-100^{\circ} \mathrm{C} ;-70{ }^{\circ} \mathrm{C} ;-35^{\circ} \mathrm{Ce}-18{ }^{\circ} \mathrm{C}$.

\section{MATERIAL E MÉTODOS}

Este trabalho foi realizado no Setor de Refrigeração e Congelamento do Laboratório de Armazenamento e Processamento de Produtos Agrícolas do Departamento de Engenharia Agrícola da Universidade Federal de Campina Grande, em Campina Grande - PB.

Para a realização deste trabalho, foram coletados frutos de cajá, junto aos produtores da região, e levados ao Laboratório onde foram selecionados quanto ao estádio de maturação. Utilizaram-se somente os frutos semimaduros, que apresentavam coloração amarela, polpa firme e isentos de manchas escuras. 
Depois do processo de seleção, $50 \mathrm{~kg}$ de cajá foram branqueados em um tanque de inox à temperatura de $60{ }^{\circ} \mathrm{C}$, por 10 minutos, para eliminação de microrganismos, e, em seguida, resfriados por 30 minutos, para atingirem a temperatura ambiente $\left(23{ }^{\circ} \mathrm{C}\right)$. Após o resfriamento, o cajá foi processado para a obtenção de sua polpa, utilizando-se de uma despolpadora Laboremus. Na Figura 1, encontram-se as diferentes etapas de processamento para a obtenção da polpa de cajá.

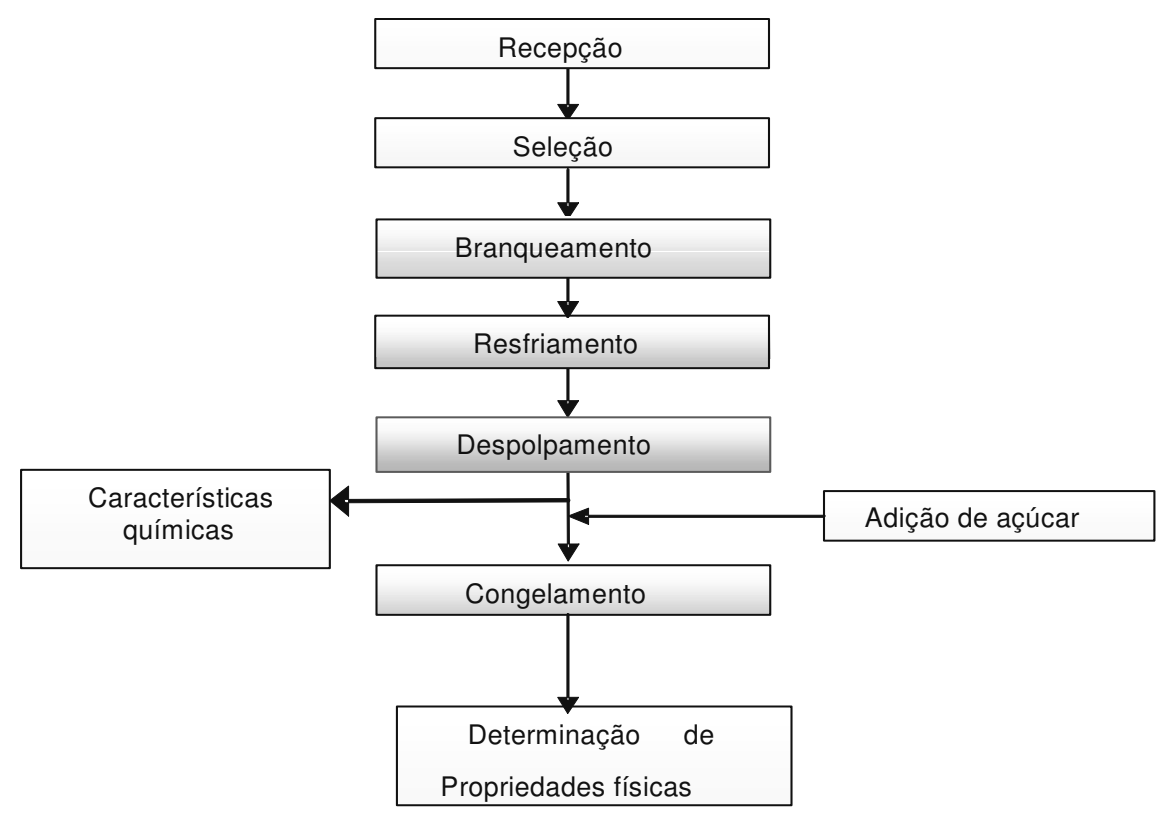

FIGURA 1. Fluxograma utilizado para o processamento da polpa de cajá.

Após o despolpamento, uma amostra de $5 \mathrm{~kg}$ foi retirada, do total obtido de $18 \mathrm{~kg}$, para sua caracterização química. Essa caracterização constou de determinação da acidez titulável, pH, umidade, sólidos solúveis totais e de açúcares totais, redutores e não-redutores.

\section{Determinações das características químicas}

Acidez titulável: sua determinação baseou-se na titulação da polpa, com solução padronizada de álcali, empregando-se a fenolftaleína como o indicador, método n⿳o 22038 da A.O.A.C. (1984).

$\mathrm{pH}$ : utilizou-se o potenciômetro Analyser, modelo pH300M.

Teor de água: determinado por meio da secagem da amostra em estufa a $105{ }^{\circ} \mathrm{C}$ até peso constante, conforme o método de $\mathrm{n}^{\mathrm{o}} 14003$ da A.O.A.C. (1984).

Sólidos solúveis totais (SST): determinação realizada por meio da leitura direta em refratômetro manual Atago e expresso em ${ }^{\circ}$ Brix.

Açúcares redutores, não-redutores e totais: o método utilizado baseia-se na redução de íons de cobre bivalente, em meio básico, pelos açúcares redutores, conforme metodologia proposta pela A.O.A.C. (1984).

Uma vez caracterizada quimicamente a polpa de cajá, o material restante foi congelado às temperaturas entre $-18{ }^{\circ} \mathrm{Ce}-196{ }^{\circ} \mathrm{C}$ por meio do criostato (Figura 2). 


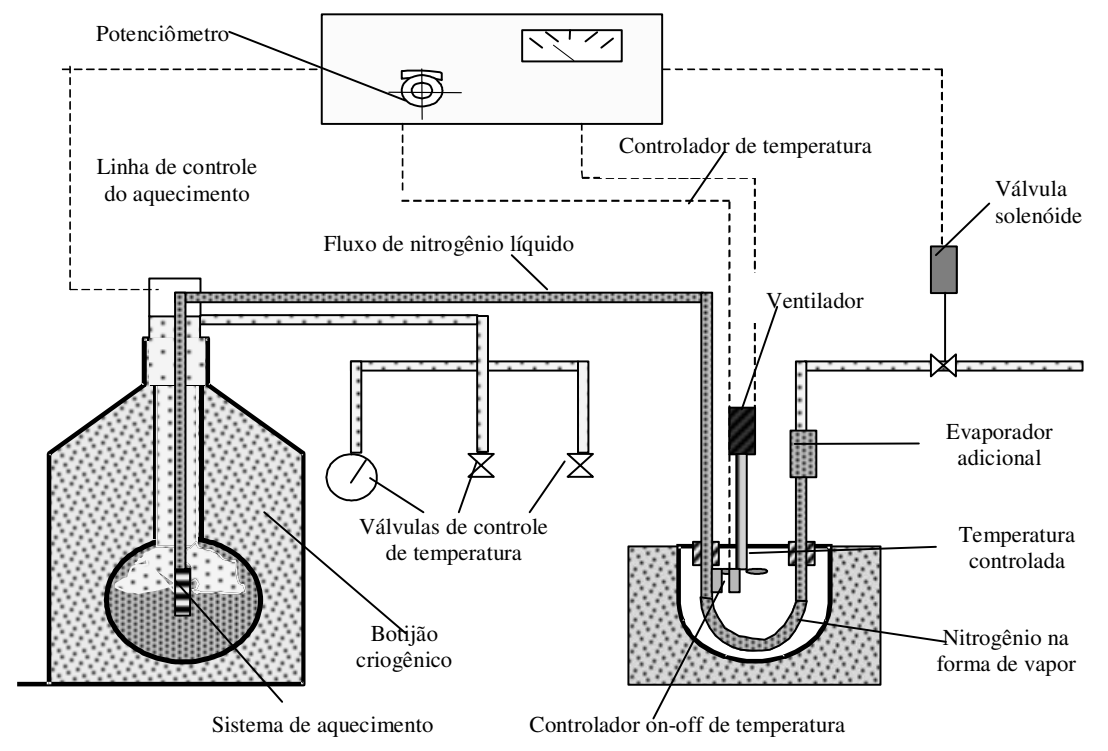

Detalhe do congelamento da amostra

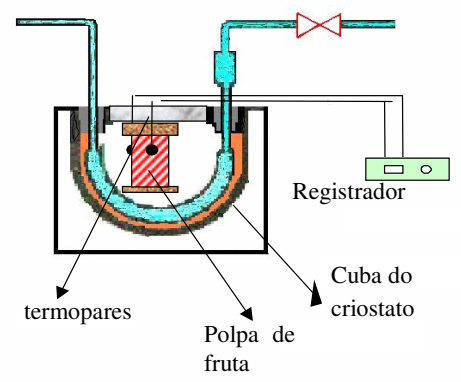

FIGURA 2. Esquema de funcionamento do criostato utilizado para congelar a polpa de cajá entre $-18{ }^{\circ} \mathrm{C}$ e $-196{ }^{\circ} \mathrm{C}$.

Para o congelamento da amostra, a cada temperatura desejada, o equipamento era ajustado por meio de um controlador de temperatura. A amostra era inserida na cuba do criostato, sendo a amostra monitorada por um termopar localizado no seu centro geométrico. Atingida a temperatura desejada, sua densidade era imediatamente determinada.

Densidade: a polpa de cajá foi congelada no interior de um plástico de alta densidade com a forma de placa plana. O volume da massa de cajá foi determinado, calculando-se o peso do deslocamento da água pela massa do produto em um recipiente com água, sem tocar sua base. Essa leitura da massa da polpa congelada de cajá submersa, medida por balança semi-analítica com precisão de $0,001 \mathrm{~g}$, menos o peso do recipiente + água, é o peso da massa de água deslocada, o qual foi usado na equação abaixo para o cálculo do volume (MOHSENIN,1978).

$$
\text { Volume }=\frac{\text { massa de água deslocada }(\mathrm{g})}{\text { massa específica da água }\left(\mathrm{g} \mathrm{cm}^{-3}\right)}
$$

Uma vez obtido o volume, a densidade foi obtida diretamente pela relação:

$$
\text { Densidade }=\frac{\text { massa da polpa congelada de cajá }(\mathrm{g})}{\text { volume da polpa de cajá }\left(\mathrm{cm}^{3}\right)}
$$

Calor específico: o calor específico dos produtos congelados foi determinado utilizando-se do método de MOLINE et al. (1961), que consistiu em se colocar a amostra no interior de um cilindro metálico, o qual foi fechado por dois tampões de borracha, um em cada extremidade, com um termopar conectado a um desses tampões de borracha para registrar a temperatura no centro geométrico do cilindro (Figura 3).

O sistema cilindro-amostra foi congelado até a temperatura negativa desejada, utilizando-se do aparelho criostato. Atingida essa temperatura, o conjunto cilindro-amostra foi colocado num bloco de poliestireno, o qual foi mantido à temperatura ambiente, registrando-se continuamente sua temperatura. $\mathrm{O}$ mesmo procedimento foi realizado com água congelada nas diferentes temperaturas negativas, sendo o calor específico determinado pelo seguinte balanço de calor: 


$$
C_{p}=\frac{M_{i} C_{p i} \frac{d T}{d \theta_{i}}-M_{a} C_{a} \frac{d T}{d \theta_{p}}}{M_{p} \frac{d T}{d \theta_{p}}}
$$

em que,

$\mathrm{M}_{\mathrm{a}}$ - massa do cilindro finito, $\mathrm{kg}$;

$\mathrm{M}_{\mathrm{i}}$ - massa do gelo, $\mathrm{kg}$;

$M_{p}$ - massa da polpa de cajá, $\mathrm{kg}$;

$\mathrm{C}_{\mathrm{a}}$ - calor específico do metal, $\mathrm{kJ} \mathrm{kg}^{-1}{ }^{\circ} \mathrm{C}^{-1}$;

$\mathrm{C}_{\mathrm{pi}}$ - calor específico do gelo, $\mathrm{kJ} \mathrm{kg}^{-1}{ }^{\circ} \mathrm{C}^{-1}$;

$\mathrm{C}_{\mathrm{p}}$ - calor específico da polpa de cajá, $\mathrm{kJ} \mathrm{kg}^{-1}{ }^{\circ} \mathrm{C}^{-1}$;

$\mathrm{d} \mathrm{T} / \mathrm{d} \theta \mathrm{i}$ - gradiente da curva de resfriamento do gelo, ${ }^{\circ} \mathrm{C} \mathrm{t}^{-1}$;

$\mathrm{d} \mathrm{T} / \mathrm{d} \theta \mathrm{p}$ - gradiente da curva de resfriamento da polpa, ${ }^{\circ} \mathrm{C}^{-1}$;

$\theta \mathrm{i}$ - tempo para o gelo, min, e

$\theta p$ - tempo para a polpa de cajá, min.

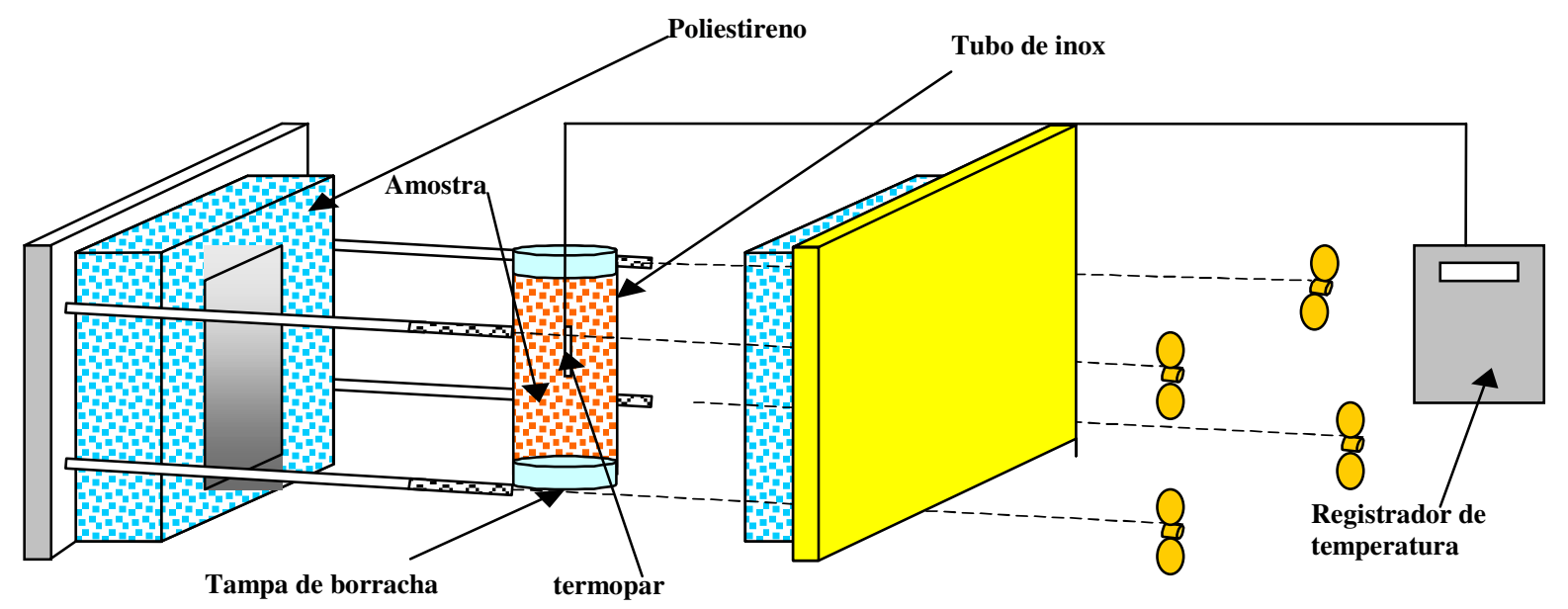

FIGURA 3. Esquema do aparelho utilizado para medir o calor específico da polpa de cajá, com base no proposto por MOLINE et al. (1961).

Para a obtenção dos dados do gelo, em cada temperatura, procedeu-se de forma similar ao descrito para a polpa, alterando-se apenas o conteúdo do cilindro, que foi feito com água destilada.

\section{Tratamento matemático}

Os dados experimentais de densidade e calor específico foram correlacionados com a temperatura, sendo os parâmetros das equações obtidos por meio de regressão não-linear, utilizando-se do programa computacional Statistica 5.0 e o Origin 5.0.

\section{RESULTADOS E DISCUSSÃO}

\section{Caracterização química da polpa de cajá}

Dependendo da época, do estádio de maturação e do local da produção, o cajá pode apresentar grande variação na sua composição. Na Tabela 1, encontram-se os valores da caracterização química da polpa utilizada neste trabalho. Como pode ser observado, o valor de sólidos solúveis totais e de acidez titulável são concordantes com os valores determinados por MORORÓ (1998), que obteve 
valores de $8,4-13,5^{\circ}$ Brix e acidez de $0,9-1,7 \%$. O pH foi maior que o observado por esse autor, que foi de 2,4 a 2,8. O teor de água encontrado experimentalmente para a polpa do cajá foi maior que os indicados por PROFRUTAS (1997), ou seja, de aproximadamente 86,90\%.

Os valores de açúcares totais, redutores e não-redutores, foram bem próximos aos valores obtidos por GONÇALVES (2000) e SILVA (2002), o que indica que a fruta estudada, em termos de seus constituintes químicos, estava dentro dos padrões.

TABELA 1. Caracterização química da polpa de cajá antes do congelamento.

\begin{tabular}{lr}
\hline Componentes & Teores \\
\hline Acidez (\% ácido cítrico) & 1,23 \\
$\mathrm{pH}$ & 4,16 \\
Teor de água (\% base úmida) & 90,06 \\
Sólidos solúveis totais ( ${ }^{\circ}$ Brix) & 9,10 \\
Açúcares totais $(\%)$ & 7,20 \\
Açúcares redutores $(\%)$ & 4,50 \\
Açúcares não-redutores $(\%)$ & 2,70 \\
\hline
\end{tabular}

\section{Densidade}

Na Tabela 2, encontram-se os dados de densidade da polpa de cajá para as temperaturas entre -18 e $-196{ }^{\circ} \mathrm{C}$. Nessa tabela, verifica-se que a densidade da polpa de cajá varia em função da temperatura e da quantidade de sólidos solúveis totais. Observa-se, também, que de $-18{ }^{\circ} \mathrm{C} \mathrm{a}-196{ }^{\circ} \mathrm{C}$ existe aumento da densidade da polpa de cajá em torno de 10,5\% para uma quantidade de sólidos solúveis totais (SST) de $9{ }^{\circ}$ Brix e de $27 \%$ para um valor de SST de $60{ }^{\circ}$ Brix.

As equações que descrevem a variação da densidade da polpa de cajá, em função das temperaturas e da quantidade de sólidos solúveis totais, encontram-se na Figura 4. Nessa figura, observa-se que a densidade obedece a uma equação exponencial com coeficiente de determinação superior a $99 \%$.

TABELA 2. Densidade da polpa de cajá em função das baixas temperaturas e dos teores de sólidos solúveis.

\begin{tabular}{crrrr}
\hline Temperaturas & \multicolumn{4}{c}{ Densidade $\left(\mathrm{kg} \mathrm{m}^{-3}\right)$} \\
\cline { 2 - 4 }$\left({ }^{\circ} \mathrm{C}\right)$ & $9{ }^{\circ}$ Brix & $20^{\circ}$ Brix & $40^{\circ}$ Brix & $60{ }^{\circ}$ Brix \\
\hline-196 & 1.017 & 1.040 & 1.100 & 1.253 \\
-180 & 988 & 1.006 & 1.069 & 1.215 \\
-140 & 968 & 989 & 1.035 & 1.124 \\
-100 & 947 & 953 & 1.013 & 1.075 \\
-70 & 935 & 947 & 992 & 1.035 \\
-35 & 924 & 938 & 981 & 1.006 \\
-18 & 920 & 931 & 960 & 986 \\
\hline
\end{tabular}

Os resultados obtidos para as temperaturas entre $-18{ }^{\circ} \mathrm{C}$ e $-35^{\circ} \mathrm{C}$ estão de acordo com os encontrados na literatura para a polpa de fruta, com teor de água semelhante, embora existam poucos trabalhos sobre a densidade da polpa de frutas, em temperaturas abaixo do ponto de congelamento e para temperaturas criogênicas, ou seja, abaixo de $-70{ }^{\circ} \mathrm{C}$, esses dados praticamente inexistem (FENNEMA \& POWRIE, 1964). KASAHARA et al. (1986) determinaram a densidade para polpa de mamão e de chirimóia para temperaturas abaixo do ponto de congelamento $\left(-18{ }^{\circ} \mathrm{C}\right)$ e encontraram valores em torno $893,43 \mathrm{~kg} \mathrm{~m}^{-3}$ e $935,2 \mathrm{~kg} \mathrm{~m}^{-3}$, respectivamente. 


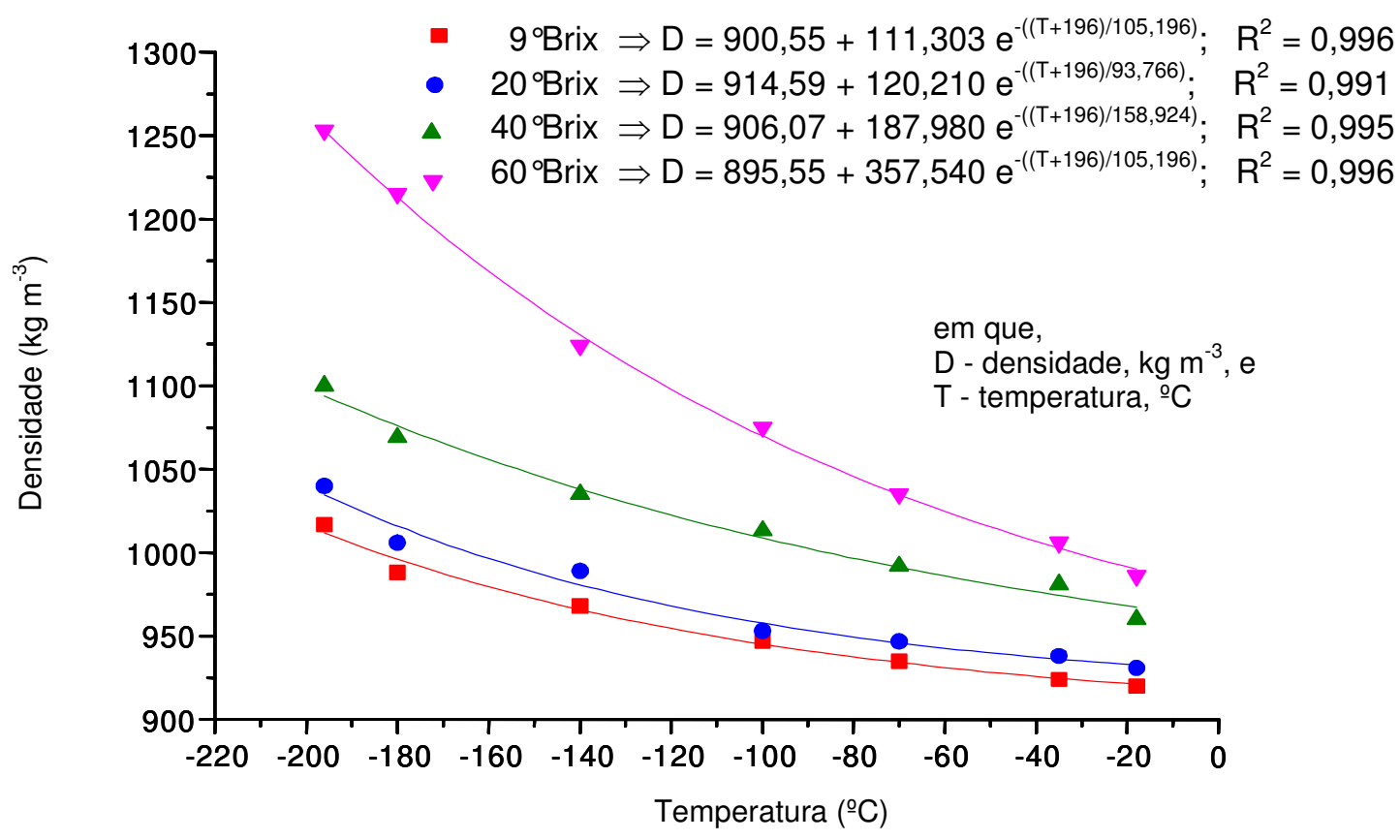

FIGURA 4. Equações da densidade da polpa de cajá em função das baixas temperaturas para os teores de sólidos solúveis totais de $9{ }^{\circ} \mathrm{Brix} ; 20^{\circ} \mathrm{Brix} ; 40^{\circ} \mathrm{Brix}$ e $60^{\circ} \mathrm{Brix}$.

\section{Calor específico}

O calor específico, como as demais propriedades termofísicas, também é influenciado pelas temperaturas abaixo do ponto de congelamento.

Nas Figuras 5 e 6, tem-se a temperatura, em função do intervalo de tempo, para a polpa de cajá e para o gelo, respectivamente, usados para a determinação do calor específico abaixo do ponto de congelamento, pela metodologia proposta por MOLINE et al. (1961).

Essas figuras indicam a determinação do calor específico da polpa de cajá, com teor de sólidos solúveis de $9{ }^{\circ}$ Brix, entre -18 a $-196{ }^{\circ} \mathrm{C}$. Devido à semelhança de execução metodológica, os gráficos correspondentes aos teores de sólidos solúveis de $20 ; 40$ e $60^{\circ}$ Brix não são mostrados, mas os resultados obtidos encontram-se na Tabela 4.

Na Figura 7, encontram-se os dados experimentais e as equações que representam a variação da temperatura com o tempo, da polpa de cajá a $9{ }^{\circ}$ Brix e do gelo, à temperatura de $-180{ }^{\circ} \mathrm{C}$. As derivadas dessas equações, em determinado intervalo de tempo, possibilitaram construir a Tabela 3 , da qual são obtidos os valores de calor específico, permitindo determinar o valor médio deste parâmetro, para a polpa de cajá à temperatura de $-180^{\circ} \mathrm{C}$ e $9^{\circ}$ Brix. 


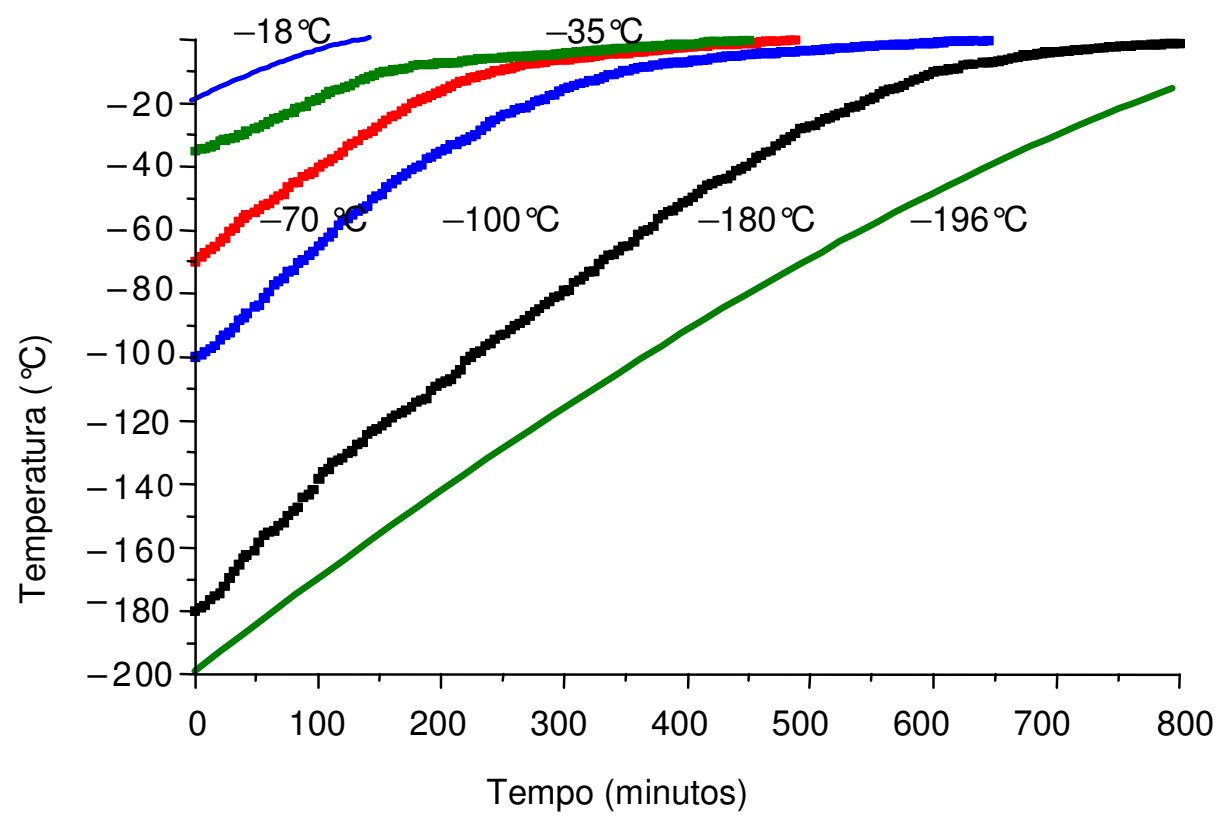

FIGURA 5. Variação da temperatura da polpa de cajá, em função do tempo, obtida no interior do equipamento confeccionado para determinar o calor específico da polpa de cajá.

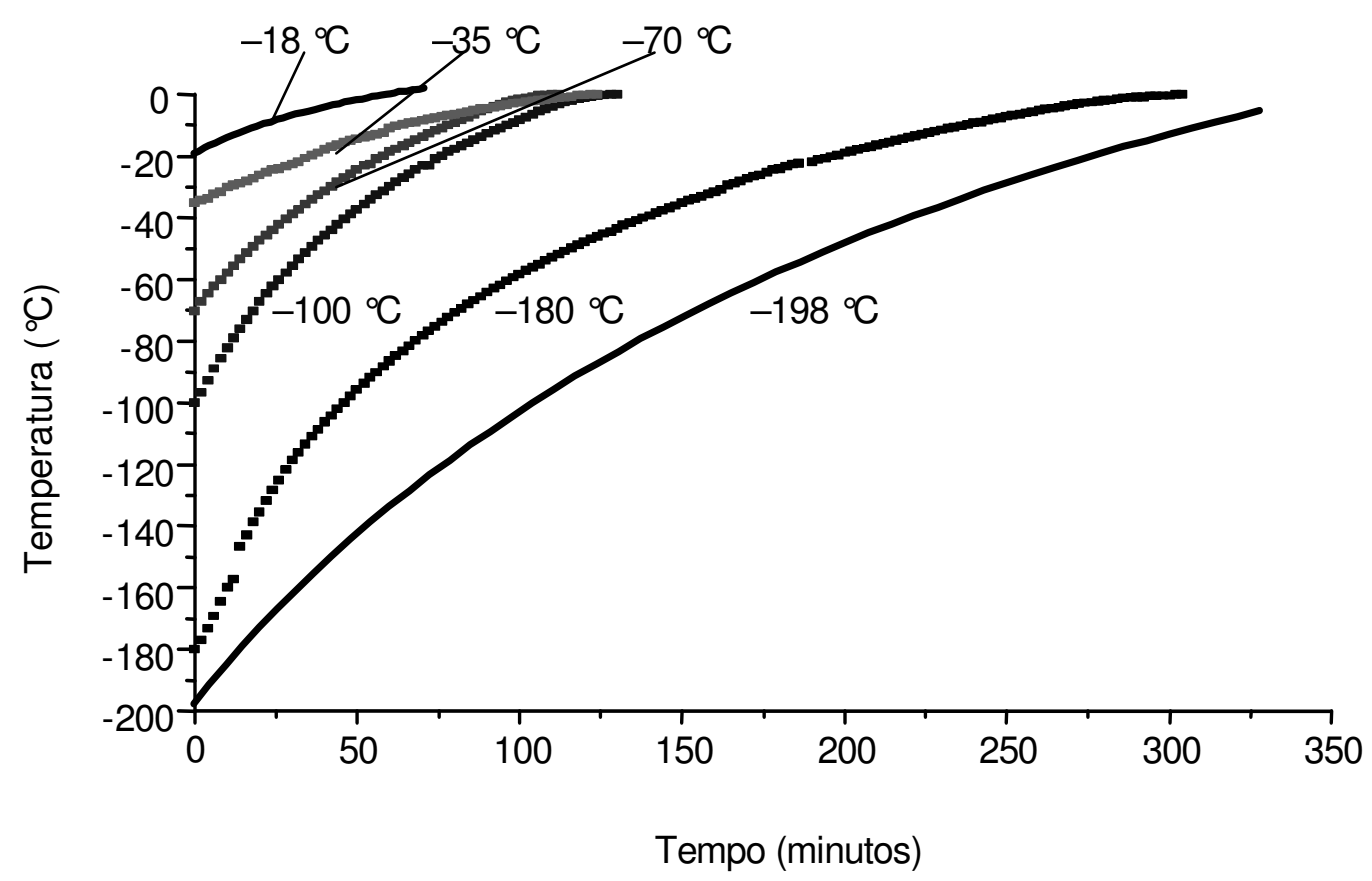

FIGURA 6. Variação da temperatura do gelo, em função do tempo, obtida no interior do equipamento confeccionado para determinar o calor específico da polpa de cajá 


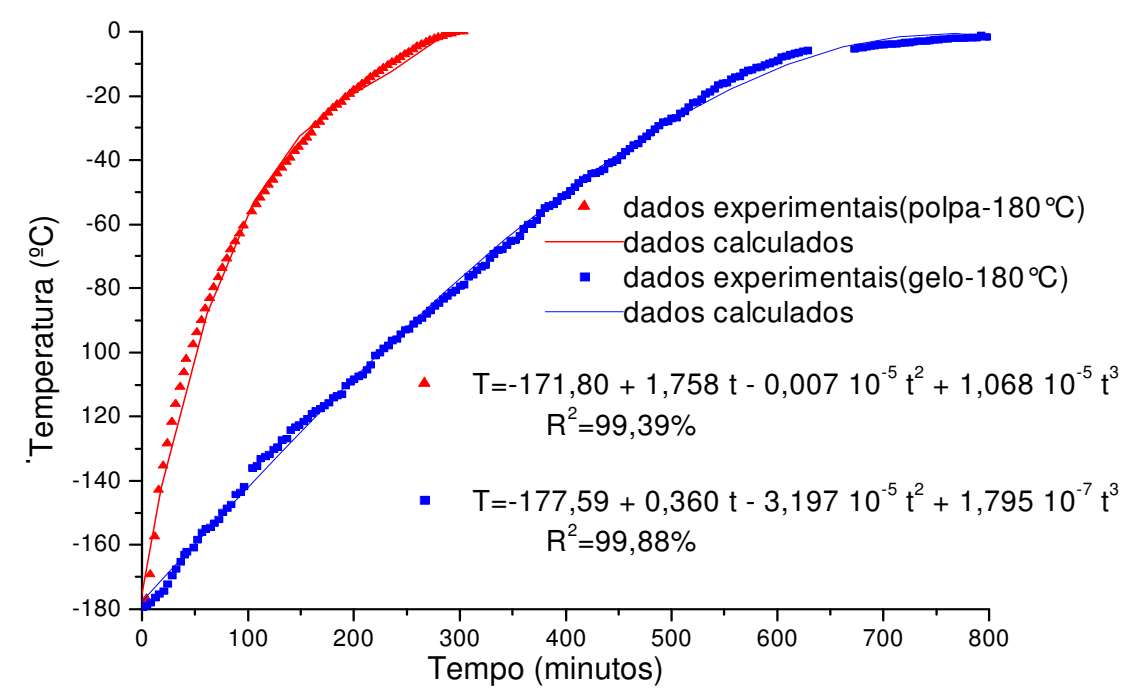

FIGURA 7. Variação da temperatura do gelo e da polpa de cajá, em função do tempo, para a determinação do calor específico $\left(-180^{\circ} \mathrm{C}\right)$.

TABELA 3. Valores para cálculo do calor específico com a polpa de cajá congelada a $-180{ }^{\circ} \mathrm{C}$.

\begin{tabular}{ccccccc}
\hline $\mathrm{T}\left({ }^{\circ} \mathrm{C}\right)$ & $\begin{array}{c}\theta_{\mathrm{i}} \\
(\mathrm{min})\end{array}$ & $\begin{array}{c}\theta_{\mathrm{p}} \\
(\mathrm{min})\end{array}$ & $\begin{array}{c}\mathrm{dT} / \mathrm{d} \theta_{\mathrm{i}} \\
\left.\left({ }^{\circ} \mathrm{C} \mathrm{min}\right)^{-1}\right)\end{array}$ & $\begin{array}{c}\mathrm{dT} / \mathrm{d} \theta_{\mathrm{p}} \\
\left.\left({ }^{\circ} \mathrm{C} \mathrm{min}\right)^{-1}\right)\end{array}$ & $\begin{array}{c}\mathrm{Cp}_{\mathrm{i}} \\
\left(\mathrm{kJ} \mathrm{kg}^{-1}{ }^{\circ} \mathrm{C}^{-1}\right)\end{array}$ & $\begin{array}{c}\mathrm{Cp}_{\mathrm{p}} \\
\left(\mathrm{kJ} \mathrm{kg}^{-1}{ }^{\circ} \mathrm{C}^{-1}\right)\end{array}$ \\
\hline-130 & 23,54 & 130,91 & 0,97 & 0,34 & 1,60 & 1,65 \\
-100 & 54,17 & 217,55 & 0,83 & 0,29 & 1,64 & 1,70 \\
-70 & 90,15 & 318,22 & 0,65 & 0,23 & 1,73 & 1,84 \\
-40 & 135,95 & 443,65 & 0,43 & 0,15 & 1,85 & 1,95 \\
\hline Valor Médio & \multicolumn{7}{c}{} & & & 1,79 \\
\hline
\end{tabular}

$\mathrm{Na}$ Tabela 4, encontram-se os valores médios do calor específico para as temperaturas entre $18{ }^{\circ} \mathrm{C}$ e $-196{ }^{\circ} \mathrm{C}$, para as polpas variando entre 9 e $60^{\circ}$ Brix. Como se observa, nessa tabela, só o valor obtido a $-35^{\circ} \mathrm{C}$, que foi de $2,41 \mathrm{~kJ} \mathrm{~kg}^{-1}{ }^{\circ} \mathrm{C}^{-1}$, é o valor que está próximo aos obtidos por KASAHARA et al. (1986), que encontraram para a polpa de chirimóia e mamão a $-35^{\circ} \mathrm{C}$, os valores de $2,42 \mathrm{~kJ} \mathrm{~kg}^{-1} \mathrm{C}^{-1}$ e $2,62 \mathrm{~kJ} \mathrm{~kg}^{-1}{ }^{\circ} \mathrm{C}^{-1}$, respectivamente. Os valores obtidos neste trabalho, para as temperaturas entre $-18{ }^{\circ} \mathrm{C}$ e $-196{ }^{\circ} \mathrm{C}$ e $9{ }^{\circ}$ Brix, variaram entre 2,485 e $1,646 \mathrm{~kJ} \mathrm{~kg}^{-1}{ }^{\circ} \mathrm{C}^{-1}$, respectivamente, e de 3,677 a 2,198 $\mathrm{kJ} \mathrm{kg}^{-1}{ }^{\circ} \mathrm{C}^{-1}$, a $60{ }^{\circ}$ Brix. Embora alguns valores estejam dentro do esperado, segundo MOLINE et al. (1961), alguns erros podem ser atribuídos ao aparelho experimental quando esse possui relação altura-diâmetro inferior a 4, pois, segundo esses autores, o cilindro só é considerado como infinito quando essa relação é superior a 4, podendo, para dimensões inferiores a 4, influenciar diretamente na curva de aquecimento. SIMÕES (1997) determinou o calor específico da polpa de manga com $93 \%$ de teor de água a $-18{ }^{\circ} \mathrm{C}$ e obteve o valor de $1,92 \mathrm{~kJ} \mathrm{~kg}^{-1}{ }^{\circ} \mathrm{C}^{-1}$, enquanto ALVARADO \& ROMERO (1989), estudando suco de melancia com 92,6\% de teor de água, encontraram calor específico na ordem de $1,97 \mathrm{~kJ} \mathrm{~kg}^{-1}{ }^{\circ} \mathrm{C}^{-1}$; no entanto, para a polpa de cajá com $9{ }^{\circ}$ Brix, o calor específico foi de $2,485 \mathrm{~kJ} \mathrm{~kg}^{-1}{ }^{\circ} \mathrm{C}^{-1}$, sendo esse bem superior às outras frutas estudadas. 
TABELA 4. Valores de calor específico da polpa de cajá em função da temperatura e do teor de sólidos solúveis, determinados pelo método de MOLINE et al. (1961).

\begin{tabular}{ccccc}
\hline \multicolumn{5}{c}{ Polpa de Cajá $\left(\mathrm{kJ} \mathrm{kg}^{-1}{ }^{\circ} \mathrm{C}^{-1}\right)$} \\
\hline Temperatura & $9{ }^{\circ}$ Brix & $20^{\circ}$ Brix & $40{ }^{\circ}$ Brix & $60{ }^{\circ}$ Brix \\
\hline-196 & 1,646 & 1,663 & 1,896 & 2,198 \\
-180 & 1,785 & 1,815 & 2,087 & 2,379 \\
-140 & 2,187 & 2,163 & 2,533 & 3,116 \\
-100 & 2,342 & 2,517 & 2,925 & 3,437 \\
-70 & 2,390 & 2,668 & 3,147 & 3,462 \\
-35 & 2,410 & 2,744 & 3,271 & 3,598 \\
-18 & 2,485 & 2,907 & 3,343 & 3,677 \\
\hline
\end{tabular}

$\mathrm{Na}$ Tabela 4, observa-se, também, que os valores do calor específico do material variaram de acordo com a temperatura de congelamento e com o teor de sólidos solúveis totais, o que é mostrado na Figura 8, juntamente com as equações que representam essas variações. Constata-se que o calor específico obedece a uma equação de segundo grau, com coeficiente de determinação superior a 0,985 e difere da equação do gelo, que se comporta como uma reta, para o intervalo de $-10{ }^{\circ} \mathrm{C}$ a $-200{ }^{\circ} \mathrm{C}$, segundo KASAHARA et al. (1986).

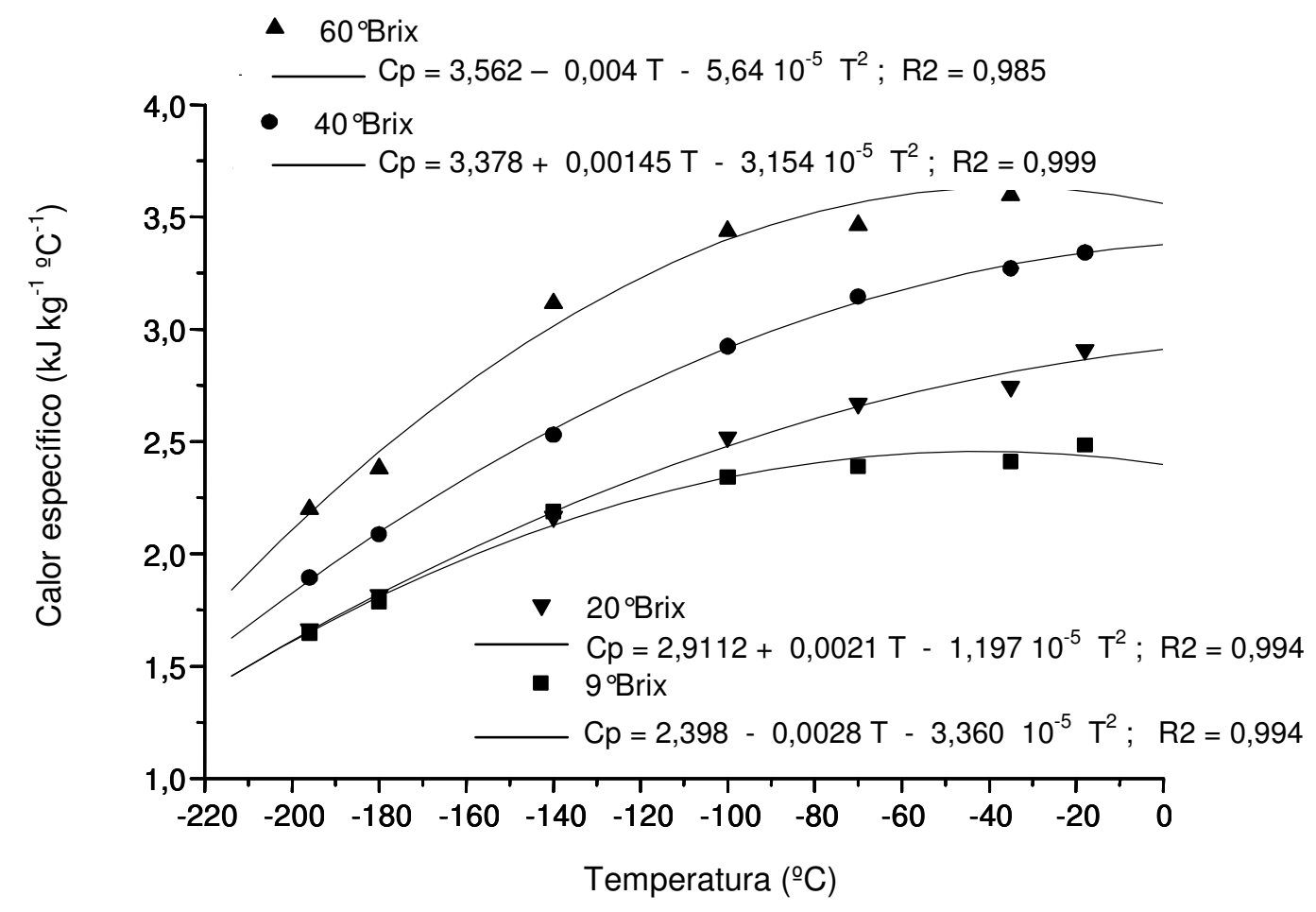

FIGURA 8. Curvas do calor específico da polpa de cajá para baixas temperaturas e diferentes teores de sólidos solúveis totais ( $9^{\circ}$ Brix; $\nabla 20^{\circ}$ Brix; • $40^{\circ}$ Brix e $\Delta 60^{\circ}$ Brix).

\section{CONCLUSÕES}

As propriedades térmicas da polpa de cajá (densidade e calor específico) são alteradas pelas temperaturas negativas e pelo teor de sólidos solúveis totais.

A densidade da polpa de cajá aumenta de $920 \mathrm{~kg} \mathrm{~m}^{-3}$ para $1.253 \mathrm{~kg} \mathrm{~m}^{-3}$ com a variação da temperatura de $-18{ }^{\circ} \mathrm{C}$ para $-196^{\circ} \mathrm{C}$ e também em função do aumento do teor de sólidos solúveis totais de $9^{\circ}$ Brix para $60^{\circ}$ Brix. 
O calor específico da polpa de cajá diminui com a redução de temperatura e aumenta com a concentração de sólidos solúveis totais, sendo o menor valor obtido de $1,646 \mathrm{~kJ} \mathrm{~kg}^{-1}{ }^{\circ} \mathrm{C}^{-1} \mathrm{a}-196{ }^{\circ} \mathrm{C} \mathrm{e}$ $9{ }^{\circ}$ Brix, e o maior de $3,677 \mathrm{~kJ} \mathrm{~kg}^{-1}{ }^{\circ} \mathrm{C}^{-1}$, para $-18{ }^{\circ} \mathrm{C}$ e $60{ }^{\circ}$ Brix.

\section{REFERÊNCIAS}

AMER, M.I.; RUBIOLO, A.C. Influencia del proceso de congelación en la variación de la vitamina C durante el almacenamiento de frutillas congeladas In: CONGRESO IBERO-AMERICANO DE INGENIERIA DE ALIMENTOS, 2., 1998, Bahía Blanca - Argentina. Anales... Bahía Blanca: Palpiqui, 1998. 1 CD ROM

ALVARADO, J.D.; ROMERO, C.H. Physical properties of fruits - 1-11: Density and viscosity of juices as functions of soluble solids and content and temperature. Latin American Applied Research, Bahía Blanca, v.19, n.24, p.15-21, 1989.

ASSOCIATION OF OFFICIAL ANALYTICAL CHEMISTS. Official Methods of Analysis. $13^{\text {th }} \mathrm{ed}$. Arlington: Williams, 1984. 1.141 p.

CIABOTTI, E.D. Alterações das propriedades físico-químicas do suco de maracujá (Passiflora edulis f. flavicarpa Deg.) para diferentes técnicas de congelamento inicial ao longo do período da armazenagem frigorificada. 2000. 107 f. Dissertação (Mestrado em Armazenamento e Processamento de Produtos Agrícolas) - Centro de Ciências e Tecnologia, Universidade Federal de Campina Grande, Campina Grande, 2000.

FENNEMA, O.; POWRIE, W. Fundamentals of low-temperature food preservation. New York: Academic Press, 1964. 478 p.

GRUDA Z.; POSTOLSKI, J. Tecnologia de la congelación de los alimentos. Zaragoza: Editorial Acribia, 1986. $631 \mathrm{p}$.

GONÇALVES, A. de L. Propriedades termofísicas de polpa de cajá (Spondias lutea L.) a baixas temperaturas. 2000. 71 f. Dissertação (Mestrado em Armazenamento e Processamento de Produtos Agrícolas) - Centro de Ciências e Tecnologia, Universidade Federal de Campina Grande, Campina Grande, 2000.

KASAHARA, I. Cinética de congelación y propriedades termofísicas en dos especies de frutales menores. In: KASAHARA, I.; GARRIDO, F.; SIMPSON, R.; ALDUNATE, M.I.; CORNEJO, F. Refrigeración y congelación de alimentos. Santiago de Chile: Maval, 1986. cap. 4, p.81-109.

MOHSENIN, N.N. Physical proprieties of plant and animal materials. New York: Gordon and Breach Science Publishers, 1978. 734 p.

MORORÓ, R.C. Como montar uma pequena fábrica de polpas de frutas. Viçosa: CPT, 1998. 68 p.

MOLINE, S.; SAWDYE, A.; SHORT, A. RINFRET,A. Thermal properties of foods at low temperature: specific heat. Food Technology, Chicago, v.15, n.5, p.228-9, 1961.

PROFRUTAS. Curso de tecnologia de industrialização de frutas. João Pessoa: Fundação de Amparo à Pesquisa do Estado da Paraíba. PROFRUTAS/SINE, 1997. 24 p.

SIEBEL, J.E. Specific heat of various products. Ice and Refrigeration, Chicago, v.2, p.256-7, 1982.

SILVA, M. Cinética de resfriamento e congelamento dos frutos de cajá (Spondias lutea L.). 2002. 100 f. Dissertação (Mestrado em Armazenamento e Processamento de Produtos Agrícolas) - Centro de Ciências e Tecnologia, Universidade Federal de Campina Grande, Campina Grande, 2002.

SIMÕES, M.R. Propriedades termofisicas da polpa de manga. 1997. 62 f. Dissertação (Mestrado em Armazenamento e Processamento de Produtos Agrícolas) - Faculdade de Engenharia de Alimentos, Universidade Estadual de Campinas, Campinas, 1997. 